\title{
Etapas do processo de identificação e conformidade dos dados do paciente em pronto
}

\section{Socorro}

\author{
Steps in the identification and compliance process of patient data in the emergency room \\ Pasos en el proceso de identificación y cumplimiento de los datos del paciente en la sala de \\ emergencias
}

Recebido: 12/01/2021 | Revisado: 12/01/2021 | Aceito: 13/01/2021 | Publicado: 14/01/2021

\author{
Suelen Cristina Zandonadi Bernal \\ ORCID: https://orcid.org/0000-0002-1327-9261 \\ Universidade Estadual de Maringá, Brasil \\ E-mail: suelenbernal_85@hotamil.com \\ Daiane Cortez Raimondi \\ ORCID: https://orcid.org/0000-0002-7143-3706 \\ Universidade Estadual de Maringá, Brasil \\ E-mail: daianetcortez@gmail.com \\ Greice Westphal \\ ORCID: https://orcid.org/0000-0001-9107-0108 \\ Universidade Estadual de Maringá, Brasil \\ E-mail: greicewes@gmail.com \\ Nelson Nardo Junior \\ ORCID: https://orcid.org/0000-0002-6862-7868 \\ Universidade Estadual de Maringá, Brasil \\ E-mail: nnjunior@uem.br \\ Carlos Alexandre Molena Fernandes \\ ORCID: https://orcid.org/0000-0002-4019-8379 \\ Universidade Estadual de Maringá, Brasil \\ E-mail: carlosmolena126@gmail.com \\ Laura Misue Matsuda \\ ORCID: https://orcid.org/0000-0002-4280-7203 \\ Universidade Estadual de Maringá, Brasil \\ E-mail: lauramisuem@gmail.com
}

\begin{abstract}
Resumo
Objetivos: Investigar se os dados de identificação das pulseiras dos pacientes de unidades de pronto-socorro correspondem com o que recomenda o Protocolo de Identificação do Paciente, e verificar se a equipe de saúde executa as etapas do processo de identificação do paciente em Unidades de Pronto Socorro. Métodos: estudo descritivo, observacional, de abordagem quantitativa e delineamento transversal, realizado de julho a setembro de 2017. A amostra constituiu-se de 240 pacientes, com mais de 24 horas de permanência na unidade de Pronto Socorro de três hospitais universitários públicos do Sul do Brasil. Os dados foram coletados em um formulário preenchido à beira do leito. $\mathrm{Na}$ análise, foi aplicado o teste qui-quadrado para as variáveis categóricas. O nível de significância adotado foi de $\alpha=0,05$. Resultados: A conformidade geral dos dados das pulseiras de identificação foi de $85,7 \%$. Dentre as etapas do processo de identificação, observou-se melhor resultado na fase "Condições de identificação" $(96,2 \%)$. Já a "Adesão à conferência dos dados de identificação" (48,3\%) foi a etapa menos executada. Conclusão: A prevalência e a conformidade dos dados de identificação do paciente mostraram-se elevadas, porém, no processo de identificação, a etapa de checagem dos dados pela equipe apresentou-se baixa.

Palavras-chave: Sistemas de Identificação de Pacientes; Segurança do Paciente; Enfermagem; Serviços Médicos de Emergência.
\end{abstract}

\begin{abstract}
Objectives: To investigate whether the identification data of patients' wristbands of emergency units correspond to what the Patient Identification Protocol recommends, and verify whether the health team performs the steps of the patient identification process at Emergency Room Units. Methods: descriptive, observational, quantitative and crosssectional study, carried out between July and September 2017. The sample consisted of 240 patients with more than 24 hours in the emergency room of three public university hospitals in the South of Brazil. Data were collected in a form filled at the bedside. In the analysis, the chi-square test was applied for the categorical variables. The level of significance was $\alpha=0.05$. Results: The overall data identification wristband compliance was $85.7 \%$. Among the
\end{abstract}


stages of the identification process, the best result occurred in the "Identification conditions" phase (96.2\%). The "Adherence to the identification data conference" (48.3\%) was the least executed step. Conclusion: The prevalence and compliance of the patient identification data were high, but, in the identification process, the team's data check phase was low.

Keywords: Patient Identification Systems; Patient safety; Nursing; Emergency Medical Services.

\section{Resumen}

Objetivos: Investigar si los datos de identificación de las pulseras de los pacientes en urgencias se corresponden con lo que recomienda el Protocolo de Identificación de Pacientes, y verificar si el equipo de salud realiza los pasos del proceso de identificación de pacientes en Unidades de Urgencias. Ayuda. Métodos: estudio descriptivo, observacional, con enfoque cuantitativo y diseño transversal, realizado de julio a septiembre de 2017. La muestra estuvo conformada por 240 pacientes, con más de 24 horas de estadía en Urgencias de tres hospitales universitarios públicos del Sur. de Brasil. Los datos se recopilaron de forma completa al lado de la cama. En el análisis se aplicó la prueba de chi-cuadrado para variables categóricas. El nivel de significancia adoptado fue $\alpha=0.05$. Resultados: El cumplimiento general de los datos de la pulsera fue del 85,7\%. Entre las etapas del proceso de identificación, se observó un mejor resultado en la fase "Condiciones de identificación" (96,2\%). La "Adherencia a la verificación de los datos de identificación” (48,3\%) fue el paso menos realizado. Conclusión: La prevalencia y conformidad de los datos de identificación del paciente fue alta, sin embargo, en el proceso de identificación, la etapa de verificación de los datos por parte del equipo fue baja.

Palabras clave: Sistemas de identificación de pacientes; Seguridad del paciente; Enfermería; Servicios médicos de emergência.

\section{Introdução}

A complexidade dos serviços de saúde e o aumento no uso de tecnologias hospitalares têm se tornado preocupação frequente das organizações de saúde que intentam prevenir ações que favorecem a oferta de assistência segura e de qualidade (Padilha et al., 2014).

A segurança é uma dimensão da qualidade que na área da saúde tem relação com a segurança do paciente. Nesse contexto, a segurança do paciente é definida como adoção de medidas que possibilitem a redução máxima de danos evitáveis ocasionados pelos cuidados em saúde (WHO, 2006). A “Agence for health care research and quality (AHRQ) conceitua segurança do paciente como ausência de potencial para ocorrência de danos ou, ainda, ausência de ocorrência de danos associados a cuidados do paciente (AHRQ, 2001).

No ano de 2007, a World Health Organization (WHO), em conjunto com a Joint Commission International (JCI), priorizou a criação de medidas de segurança do paciente, por meio de nove soluções para prevenção de erros e eventos adversos na assistência à saúde e, dentre elas, consta a Identificação do Paciente (WHO, 2003).

Considerada de extrema relevância dentre as nove soluções propostas, a identificação do paciente é prioritária em instituições de saúde, pois tem como objetivo garantir que o procedimento/cuidado seja executado no paciente certo, evitandose eventos adversos no processo de cuidado (Brasil, 2013; Hoffmeister \& Moura, 2015).

A identificação incorreta do paciente pode resultar em eventos adversos graves tais como: erros de medicação, erros na administração de hemocomponentes, erros na entrega de diagnósticos, procedimentos realizados em pacientes errados e/ou em locais errados da região corpórea, entrega de recém-nascidos a famílias erradas, dentre outros (WHO, 2003).

O processo de identificação do paciente é um componente essencial e viável nos processos assistenciais de serviços de saúde por ser considerada uma medida de baixo custo e passível de prevenir eventos adversos evitáveis (Bates et al., 2009; Latham et al., 2012). Diante disso, a WHO propôs medidas normativas, com finalidade de minimizar a ocorrência de eventos adversos associados à identificação do paciente, tais como: uso de pulseira desde a admissão até a alta (que deve ser conferida antes da realização de qualquer procedimento), criação de protocolos institucionais, e envolvimento do paciente/família no processo de identificação (WHO, 2003). 
Em que pese o fato de o uso da pulseira ser indicado como melhor forma de identificar o paciente, a conferência dos dados nas pulseiras, antes da realização de cuidados/procedimentos, ainda não é prática comum entre os profissionais de saúde (Prade Hemesath et al., 2015). Estudo realizado em um hospital universitário da cidade de Porto Alegre, com objetivo de analisar o impacto de ações educativas nos resultados do indicador de adesão à verificação da pulseira de identificação de pacientes, antes da realização de cuidados de maior risco, constatou, que antes de realizar às ações educativas, a prevalência de não adesão à conferência da pulseira era de 57,1\% (Prade Hemesath et al., 2015).

No contexto hospitalar, a identificação é considerada crucial, especialmente em unidades de Pronto Socorro, porque esse setor possui peculiaridades que elevam o risco de danos ao cuidado em saúde, como elevada demanda de pacientes, gravidade dos casos, sub-dimensionamento da equipe de profissionais, dentre outros (Brasil, 2006; Souza et al., 2011; Zanelatto \& Dal Pai, 2010).

Além da identificação propriamente dita, é importante que os profissionais cumpram as etapas do processo de identificação que, de acordo com o Protocolo de Identificação do Paciente (Brasil, 2013), compõem-se de: presença de recurso identificador; verificação dos dados de identificação antes da realização de qualquer procedimento; componentes da identificação (nome, sobrenome, número de registro, etc); condições do recurso identificador (ausência de falhas na impressão, sujidade, isento de rasgo, corte, dobradura ou problema no fecho/adesivo, tamanho adequado) (Brasil, 2013).

Os quesitos referidos são necessários porque a identificação não se resume apenas em identificar, mas, também, na realização de todo o processo de identificação para que, a partir disso, seja, assim, capaz de prevenir falhas nas diversas esferas da prática do cuidado (Tase \& Tronchin, 2015).

Com base no exposto, questiona-se: os dados de identificação das pulseiras de pacientes atendidos em PS de hospitais universitários têm conformidade com o que preconiza o Protocolo de Identificação do Paciente (Brasil, 2013)? A equipe de saúde desse setor realiza as etapas do processo de identificação?

Para responder a essas questões, o presente estudo teve como objetivos: investigar se os dados de identificação das pulseiras de pacientes de unidades de pronto-socorro correspondem com o que recomenda o Protocolo de Identificação do Paciente (Brasil, 2013), e verificar se a equipe de saúde realiza as etapas do processo de identificação do paciente em unidades de pronto-socorro.

\section{Metodologia}

Estudo descritivo, observacional, de abordagem quantitativa e delineamento transversal, realizado em três Hospitais Universitários da região Sul do Brasil, representados pelas siglas HA, HB e HC.

Utilizou-se amostragem não probabilística, cujos participantes foram selecionados por conveniência. O tamanho da amostra foi calculado a partir do número de internações do primeiro trimestre do ano de 2016 por estabelecimento de saúde. As informações foram obtidas do site DATASUS. O tamanho da amostra foi de 240 indivíduos, assim distribuídos: 61 do HA; 91 do $\mathrm{HB}$ e 88 do HC.

Os participantes foram todos os pacientes com tempo de permanência igual ou superior a $24 \mathrm{~h}$ no PS e em condições de verbalizar sobre o assunto questionado. Esse quesito foi verificado por meio da aplicação da Escala de Glasgow, que avalia o nível de consciência do entrevistado.

As entrevistas foram realizadas em pacientes com Glasgow entre 13 e 15 pontos, os quais são obtidos através da observação do paciente, atribuindo um valor a cada parâmetro observado (abertura ocular, resposta verbal e resposta motora), sendo a soma dos pontos atribuídos a cada parâmetro o resultado da escala (Teasdale Graham et al., 2014).

A coleta de dados foi realizada por meio de observação e entrevista estruturada, no período de julho a setembro de 
2017, utilizando-se um formulário de entrevista, composto pelas seguintes etapas a serem avaliadas no processo de identificação: presença de identificação, adesão à conferência dos dados de identificação do paciente, componentes da identificação, e condições da identificação.

Os dados foram agrupados em banco de dados no Excel 2010 (Microsoft Inc.) e, posteriormente, analisados no programa SPSS (Statistical Package for the Social Sciences), versão 22.0 (Andy Field, 2009). Para comparar possíveis diferenças entre os hospitais, foi aplicado o teste qui-quadrado para as variáveis categóricas. Para todas as análises realizadas, adotou-se nível de significância de $\alpha=0,05$.

Neste estudo, "conformidade" foi considerada como o atendimento aos requisitos determinados pelo Protocolo de Identificação do Paciente ${ }^{6}$ e como "não conformidade", qualquer situação que indicasse o contrário.

Esta pesquisa atendeu aos princípios éticos da Resolução nº66/12, do Conselho Nacional de Saúde e está registrado Comitê Permanente de Ética em Pesquisas Envolvendo Seres Humanos (COPEP), da Universidade Estadual de Maringá (UEM), sob parecer n ${ }^{\circ}$ 2.144.505, CAAE 65459617.7.0000.0104, JUN/2017.

\section{Resultados}

Na Figura 1, constam os resultados da conformidade e não conformidade dos dados de identificação das pulseiras dos pacientes.

Figura 1. Conformidade e não conformidade geral dos dados das pulseiras de identificação de pacientes em prontos socorros de hospitais universitários. Sul do Brasil, 2017.

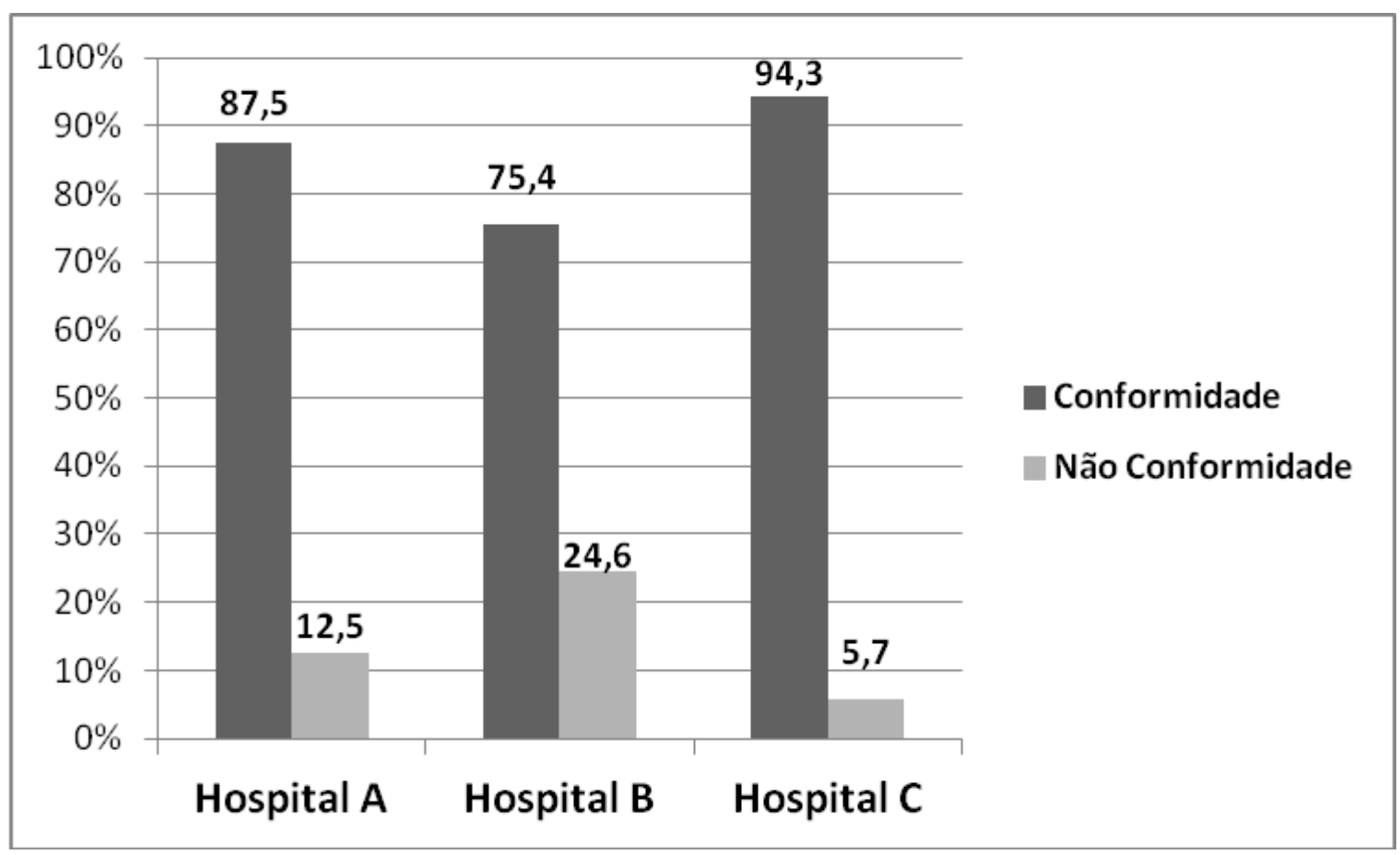

Fonte: Autores.

Os dados da Tabela 1 referem-se à execução e não execução das etapas do processo de identificação do paciente pela equipe de saúde. Foi possível observar que a prevalência de execução da etapa 2 (adesão à conferência) foi significativamente inferior às demais etapas do processo de identificação. 
Tabela 1. Conformidade nas etapas de avaliação da identificação do paciente em pronto-socorro de hospitais universitários do Paraná, 2017.

\begin{tabular}{ccccc}
\hline Conformidade & $\begin{array}{c}\text { TOTAL }(\mathbf{n = 2 4 0}) \\
\mathbf{n}(\boldsymbol{\%})\end{array}$ & $\begin{array}{c}\text { HA (n=61) } \\
\mathbf{n}(\boldsymbol{\%})\end{array}$ & $\begin{array}{c}\text { HB }(\mathbf{n}=\mathbf{9 1}) \\
\mathbf{n}(\boldsymbol{\%})\end{array}$ & $\begin{array}{c}\text { HC }(\mathbf{n}=\mathbf{8 8}) \\
\mathbf{n}(\boldsymbol{\%})\end{array}$ \\
\hline Presença de identificação & $209(87,1)$ & $48(78,7)$ & $73(80,2)$ & $88(100,0)^{*}$ \\
Adesão à conferência & $110(45,8)$ & $13(27,1)$ & $29(40,3)$ & $68(77,3)^{*}$ \\
Componentes de identificação & $180(86,2)$ & $42(87,5)$ & $55(75,4)$ & $83(94,3)^{\#}$ \\
Condições da identificação & $201(96,2)$ & $45(93,7)$ & $68(93,1)$ & $88(100,0)$ \\
\hline
\end{tabular}

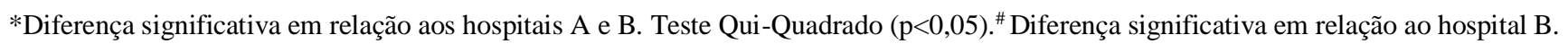
Teste Qui-Quadrado $(\mathrm{p}<0,05)$.

Fonte: Autores.

\section{Discussão}

O cuidado seguro é inerente à identificação adequada do paciente, mas, para que isso ocorra, é necessário que os pacientes utilizem pulseira de identificação, a qual deve conter informações corretas, como por exemplo: nome completo, data de nascimento e número de registro (Brasil, 2013).

Além disso, para que o processo de identificação ocorra de forma integral e sistematizada, é essencial que as condições do recurso identificador (no caso do presente estudo, as pulseiras) estejam de acordo com o preconizado no Protocolo de Identificação do Paciente: ausência de falhas na impressão, sujidade, isento de rasgo, corte, dobradura ou problema no fecho/adesivo, tamanho adequado (Brasil, 2013), e que o profissional realize a conferência dos dados de identificação antes de realizar qualquer procedimento no paciente (Brasil, 2013; WHO, 2003).

Os dados obtidos no presente estudo (Figura 1), referentes à conformidade da pulseira de identificação nas três instituições, evidenciam a preocupação com o cuidado de qualidade e a promoção da segurança do paciente, uma vez que o percentual foi maior que $70 \%$ em todas instituições, com destaque para o Hospital C, que se aproximou de $95 \%$.

Corroborando com o dado do presente estudo, uma pesquisa norte americana, realizada entre 1999 e 2000, avaliou as pulseiras de identificação em 217 instituições de saúde e constatou que, dentre as 1.757 .730 pulseiras analisadas, 45.197 $(2,57 \%)$ apresentavam problemas como: ausência de pulseira (71,6\%); ilegibilidade dos dados (7,7\%); informações incorretas $(6,8 \%)$; borrões/ rasuras $(9,1 \%)$; dados conflitantes $(3,7 \%)$; e pulseira em paciente errado (1,1\%) (Howanitz et al., 2002$)$.

Observa-se na Tabela 1 que, na etapa presença de identificação, a ausência de pulseiras foi de 12,9\%, demonstrando que as estratégias para promoção e manutenção da identificação adequada devem ser reforçadas.

No que tange a relação custo-efetividade da identificação do paciente, uma auditoria realizada em 89 instituições de saúde de cuidados intensivos, em oito países europeus (Bélgica, República Checa, França, Irlanda, Países Baixos, Polônia, Espanha e Reino Unido), evidenciou que a identificação do paciente é uma estratégia básica, com elevada efetividade na redução de falhas, mas pouco executada (Suñol et al., 2009).

Quando avaliado o processo de identificação (tabela 1), observa-se que o maior percentual de execução ocorreu na etapa 4 (Condições da identificação), o que demonstra a preocupação das instituições na escolha do material utilizado para a confecção e impressão das pulseiras, facilitando a integridade e legibilidade dos dados de identificação. Já o menor percentual 
de execução foi observado na segunda etapa (Adesão à conferência dos dados de identificação $=48,3 \%$ ), o que evidencia o despreparo e, talvez, desconhecimento dos profissionais de saúde acerca da relevância desta etapa do processo de identificação.

Corroborando com a afirmação anterior, uma pesquisa com objetivo de verificar a opinião e a prática dos profissionais de saúde sobre identificação do paciente evidenciou que $17,1 \%$ dos profissionais desconheciam o motivo do uso da pulseira e 40,7\% referiram que o uso da pulseira era dissociável do cuidado seguro (Martínez-Ochoa et al., 2010).

Os resultados deste estudo indicam a necessidade de reforçar as práticas referentes à identificação do paciente, pois foi constatado que as etapas do processo de identificação não são executadas de forma integral e sistematizada pelos profissionais de saúde, e isso, certamente, conduz a riscos advindos da identificação incorreta dos usuários, os quais culminam, na maior parte das vezes, em agravos à saúde.

\section{Conclusão}

O presente estudo constatou que a prevalência da conformidade dos dados da pulseira de identificação foi elevada quando considerada amostra total, ressaltando-se que o $\mathrm{HC}$ aumenta a porcentagem de conformidade dos dados de identificação de HA e HB. Entretanto, ao considerar o processo de identificação, verificou-se baixa prevalência de adesão à verificação dos dados da pulseira pelos profissionais de saúde nas três instituições estudadas.

Apesar de este estudo ter sido realizado em três instituições, considera-se como limitação o fato de ter sido realizado em apenas um tipo de serviço (Pronto Socorro) e em instituições públicas, o que não permite generalizações. Sugerem-se, então, mais estudos sobre identificação do paciente, com ênfase em ações educativas e estratégias de fomento à sua efetividade.

\section{Referências}

AHRQ, A. for H. R. and Q. (2001). Annual Report on Research and Management. Florida Fish and Wildlife . Conservation Com-mission. http://www.ahrq.gov/about/annrpt01/

Andy Field. (2009). Discovering Statistics using SPSS Statistics. SAGE Publications, 66, 822. http://www.amazon.com/Discovering-Statistics-using-IBMSPSS/dp/1446249182

Bates, D. W., Larizgoitia, I., Prasopa-Plaizier, N., \& Jha, A. K. (2009). Global priorities for patient safety research. BMJ (Online), 338(7705), 1242-1244. https://doi.org/10.1136/bmj.b1775

Brasil, M. da S. (2006). Política Nacional de Atenção as Urgências. (M. da Saúde (org.); $3^{\circ}$ ed).

Brasil, M. da S. (2013). Agência Nacional de Vigilância Sanitária. Portaria no 36, de 25 de julho de 2013. Institui ações para a segurança do paciente em serviços de saúde e dá outras providências.

Hoffmeister, L. V., \& Moura, G. M. S. S. de. (2015). Use of identification wristbands among patients receiving inpatient treatment in a teaching hospital. Revista Latino-Americana de Enfermagem, 23(1), 36-43. https://doi.org/10.1590/0104-1169.0144.2522

Howanitz, P. J., Renner, S. W., \& Walsh, M. K. (2002). Continuous Wristband Monitoring Over 2 Years Decreases Identification Errors. Archives of Pathology \& Laboratory Medicine, 126(7), 809-815. http://www.archivesofpathology.org/doi/abs/10.1043/0003$9985 \% 282002 \% 29126 \% 3 \mathrm{C} 0809 \% 3 \mathrm{ACWMOYD} \% 3 \mathrm{E} 2.0 . \mathrm{CO} \% 3 \mathrm{~B} 2$

Latham, T., Malomboza, O., Nyirenda, L., Ashford, P., Emmanuel, J., M'baya, B., \& Bates, I. (2012). Quality in practice: Implementation of hospital guidelines for patient identification in Malawi. International Journal for Quality in Health Care, 24(6), 626-633. https://doi.org/10.1093/intqhc/mzs038

Martínez-Ochoa, E. M., Cestafe-Martínez, A., Soledad Martínez-Sáenz, M., Belío-Blasco, C., Caro-Berguilla, Y., \& Rivera-Sanz, F. (2010). Evaluación de la implantación de un sistema de identificación inequívoca de pacientes en un hospital de agudos. Medicina Clínica, 135, 61-66. https://doi.org/10.1016/S00257753(10)70022-1

Padilha, E. F., Haddad, M. D. C. F. L., \& Matsuda, L. M. (2014). Qualidade Dos Registros De Enfermagem Em Terapia Intensiva: Avaliação Por Meio Da Auditoria Retrospectiva. Cogitare Enfermagem, 19(2). https://doi.org/10.5380/ce.v19i2.32103

Prade Hemesath, M., Barreto dos Santos, H., Schroder Torelly, E., Bolzan Motta, M., Silveira Pasin, S., \& Muller de Magalhães, A. (2015). Avaliação e gestão da adesão dos profissionais à verificação da identificação do paciente. Revista Acreditação, 5(9), 45-54.

Souza, C. C. de, Toledo, A. D., Tadeu, L. F. R., \& Chianca, T. C. M. (2011). Classificação de risco em pronto-socorro: concordância entre um protocolo institucional brasileiro e Manchester. Revista Latino-Americana de Enfermagem, 19(1), 26-33. 
Research, Society and Development, v. 10, n. 1, e33910111923, 2021 (CC BY 4.0) | ISSN 2525-3409 | DOI: http://dx.doi.org/10.33448/rsd-v10i1.11923

Suñol, R., Vallejo, P., Groene, O., Escaramis, G., Thompson, A., Kutryba, B., \& Garel, P. (2009). Implementation of patient safety strategies in European hospitals. Quality and Safety in Health Care, 18(SUPPL. 1). https://doi.org/10.1136/qshc.2008.029413

Tase, T. H., \& Tronchin, D. M. R. (2015). Sistemas de identificação de pacientes em unidades obstétricas e a conformidade das pulseiras. Acta Paulista de Enfermagem, 28(4), 374-380. https://doi.org/10.1590/1982-0194201500063

Teasdale Graham, Allan, D., Brennan, P., McElhinney, E., \& Mckinnon, L. (2014). Forty years on: updating the Glasgow Coma Scale. Nursing Times, $110(42), 12-16$

WHO, W. H. O. (2003). Patient Identification: The Foundation for a Culture of Patient Safety. Journal of Nursing Care Quality, 18(1), 73-79. https://doi.org/10.1097/00001786-200301000-00010

World Health Organization WHO. (2006). Quality of care: A process for making strategic choices in health systems. The Journal of the American Medical Association, 267, 1-50. https://apps.who.int/iris/bitstream/handle/10665/43470/9241563249_eng.pdf\%0Afile:///E:/THESIS WENNY/Jurnal/Quality of Care_B.Def WHO.pdf

Zanelatto, D. M., \& Dal Pai, D. (2010). Práticas de acolhimento no serviço de emergência: a perspectiva dos profissionais de enfermagem. Ciência, Cuidado e Saúde, 9(2). https://doi.org/10.4025/cienccuidsaude.v9i2.9390 